\title{
Chronology of the Funnel Beaker Culture Settlement in Western Ukraine in the Context of Radiocarbon Dating
}

\section{Małgorzata Rybicka ${ }^{a}$}

\begin{abstract}
The article concerns the absolute dating of the Funnel Beaker culture over the upper Bug and the upper Dniester (Polish-Ukrainian borderland and western Ukraine). Also discussed are the relations of the community of this culture from the eastern zone of the south-eastern group with the Tripolye culture.
\end{abstract}

KEY-WORDS: Funnel Beaker culture, Tripolye culture, Western Ukraine.

\section{INTRODUCTION}

It has been 50 years since the publication of the work of Jan Kowalczyk (1969) under the title Poczatti neolitu na ziemiach polskich [The Origins of the Neolithic Age on Polish Territories]. The researcher referred in his work to the concepts of the genesis of the Funnel Beaker culture (FBC) generally accepted at that time, noting that the influences in the formation of the European Neolithic from the southeast, between the Carpathians and the Black Sea, have been underestimated. The common opinion about the very late chronology of the Funnel Beaker culture in its southeastern area is a further confirmation of this fact [...] (Kowalczyk 1969: 59). Taking into account the very early radiocarbon determination that had been obtained for a sample from Gródek on the Bug, Hrubieszów district, which he associated with the FBC (Kowalczyk 1968; 1969: 36), he stressed the importance of the upper Bug basin as a place that should be taken into account when considering the initiation of this cultural phenomenon. In his opinion, [...] the emergence of the Tripolye culture in a large area between the Carpathians and the Dnieper [...] indicates that the Middle Eastern impacts were spreading from a powerful front facing north (Kowalczyk 1969: 59). In addition, he believed that there had also

a Institute of Archaeology, Rzeszów University, io Moniuszki st., 35-oIs Rzeszów, Poland, e-mail: mrybicka@interia.eu 
I04 $\mid$ Matgorzata Rybicka

been close contacts between the FBC and the Tripolye culture (TC), and the early dates obtained for the already well-developed "beaker"-materials from Gródek allowed them, and also the beginning of the FBC, to be assigned to an earlier period (Kowalczyk 1969: 60).

The matter of the mutual relationships between the western world, represented by the FBC communities, and the TC has been repeatedly raised in Polish, Russian and Ukrainian literature during the last 50 years (Zaharuk [Захарук] 1959; Zbenovich [Збенович] 1976; Balcer 198I; Kośko 198г; Movsha [Мовша] 1985; Videiko 2000; Rybicka 2017). On the other hand, studies on the chronology of the FBC in Western Ukraine have not yet been undertaken. The long term research conducted there by Mykola Peleshchyshyn of settlements of this culture, such as the Lysivka site, Vinnyky, Lviv Raion, the Tshub site at Lezhnitsa, Ivaniche Raion, and at Tadani, KamiankaBuzka Raion (Peleshchyshyn [Пелещишин] 1990; 2004) have not yet become widely known in the scientific literature. This was probably the result of publishing only short reports in which only some of the results of fieldwork were presented. Until recently, no radiocarbon analyses have been carried out on samples from the known sites of the FBC, except for the settlement in Zimne (Bronicki et al. 2003).

As part of a project carried out at the Institute of Archaeology of the University of Rzeszów entitled Studies on the eastern borderland of the Central European cultural province in prehistory and early Middle Ages, headed by Andrzej Pelisiak, and the field director Jan Machnik, limited archaeological excavations have been carried out on the FBC settlement at the site Grodzisko (Horodyshche) III in Kotoryny on the upper Dniester (Hawinskyj et al. 20I3). Their results showed the problems of this culture in Western Ukraine in a new light, both in terms of its chronology and spatial extent (Rybicka 20I7).

The results obtained then, as well as the results of research carried out in the following years in the borderlands of the territories of the FBC and the TC in western Volhynia (Rybicka 2017), resulted in the next project (NCN Opus $8 \mathrm{UMO} 2014 / 15 / \mathrm{B} /$ $\mathrm{HS}_{3}$ /02486): Between the East and the West. Dynamics of Social Changes from the Eastern Carpathians to the Dniepr in the 4 th - beginning of the 3rd Millennium BC. One of its main goals was to determine the character and chronology of contacts between these cultures.

The tasks undertaken in this project, still in progress at the time of writing, have highlighted the importance of the FBC and its contacts with the TC (Rybicka 20I7), and also indicated the importance of the absolute dating of the cultural phenomena taking place there (Rybicka et al. 2019). It is particularly important to determine the time of appearance and disappearance of the "Beaker People" communities in western Ukraine.

According to Sławomir Kadrow (2005: 13), the functioning of the first settlement phase of the FBC in Zimne can be dated between 3650-3400 BC, i.e. a similar period 
as the first stage of settling by these communities on the Bug river, such as in Gródek, Hrubieszów district (Włodarczak 2006: 5I). This researcher assigns the second phase in Zimne to the period 3050-2600 BC. Piotr Włodarczak (2006: 5I), however, dates it to $3100-2800 \mathrm{BC}$. This dating diverges to a considerable extent from the adopted time frames for the functioning of the second stage of the settlement of Gródek, which is assigned to the period 3400-3100 BC (Włodarczak 2006: 5I). These discrepancies show the difficulties in determination of the dating the later stages of the $\mathrm{FBC}$ in the Bug river region.

The re-analysis of the radiocarbon dates received for the eastern and south-eastern group of the $\mathrm{FBC}$ is an important element of discourse in recent years in the literature. The works of Marek Nowak (2009), Piotr Włodarczak (2006), Janusz Kruk and Sarunas Milisauskas (2018) are part of this trend. In this connection, we should revisit the results of radiocarbon dating received for the FBC from western Ukraine.

CRITICAL ANALYSIS OF THE AVAILABLE RADIOCARBON DATES FOR THE FUNNEL BEAKER CULTURE SETTLEMENTS FROM WESTERN UKRAINE

Lack of good starting materials for radiocarbon dating from previously researched sites, such as Mali Hrybovychi, Zhovkva Raion (Havinskyi [Гавінський] 2009), Rudniki, Mykolaiv Raion or the Lysivka site at Vynnyky, (Havinskyi, Pastekevich [Гавінський, Пастеркєвіч] 20I6), hinders situating in time the functioning the FBC communities in western Ukraine. That is why such a large significance is now attributed to the series of dates obtained in the I990s from the site in Zimne (Bronicki et al. 2003), and in recent years from the settlements of the Grodzisko (Horodyshche) III site at Kotoryny. In addition, we have single $\mathrm{I}_{4} \mathrm{C}$ determinations for the settlement in the Lysivka site at Vynnyky, and for the FBC from the site Podobanka at Novomalin, Ostroh Raion (Hawinskyj et al. 2013; Rybicka 20I7). The quoted sites represent several different regions of the oecumene of this culture: the area on the upper Dniester, on the upper Bug, the eastern Roztocze and western Volhynia.

\section{Zimne, Volodymyr-Volynskyi Raion}

In the case of the site in Zimne, Volodymyr-Volynskyi Raion, I2 radiocarbon determinations made with the scintillation method from animal bones were associated with material of the FBC (Bronicki et al. 2003: 33). Several dates obtained from the samples taken from the floors of the pits No. 30/97 and 8/97 correspond to the classical stage of this culture. They are respectively: $4920 \pm 50 \mathrm{BP}(\mathrm{Ki}-6873), 4770 \pm 60 \mathrm{BP}$

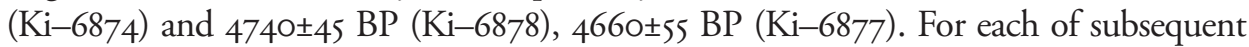
features (No. 2/97, 5/97, 3/97) also two differing from each other dates were obtained, made from various samples. The following results were obtained: feature No. 2/97: 4390 55 BP 
Io6 $\mid$ Matgorzata Rybicka

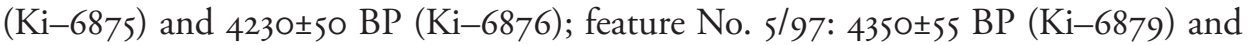

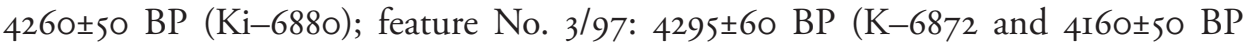
(Ki-687I). In the case of features No. 2/97 and 5/97, the samples analysed came from their upper parts (Bronicki et al. 2003; Rybicka et al. 2019). Some dates were obtained from samples coming from the upper fills of shallow pits, including feature No. 3/97 and subsequent ones, with numbers: $3 \mathrm{I} / 97$ and 32/97, for the latter they are: $4 \mathrm{I} 2 \mathrm{O} \pm 50 \mathrm{BP}$ (Ki-6870) and 4080 55 BP (Ki-6869).

In the back-filled depressions of archaeological features, are often deposited materials originating from another phase of the site not corresponding to the date of the feature itself (Kadrow 199I), which reduces the value of dates obtained from samples taken from these places. The dating results of organic material from pits 31/97 and $32 / 97$ therefore seem to be debatable, the more so because they do not correspond to the result of the archaeological analysis of pottery - they are too late in relation to this. Of particular concern are the very late results obtained for features 31/97 and 32/97, whose context is not certain. It can be assumed that they do not refer to the FBC.

Sławomir Kadrow, justifying the discrepancy between the dating of the second settlement phases of the FBC in Gródek and Zimne, links it with the regional diversity of this culture, following the classical period (Kadrow 2005: 13).

It is possible to assign the identified imports found in deposits of the second settlement phase in Gródek with those characteristics of the Gordineşti group (Dergachev [Дергачев] 1980; Sirbu [Сырбу] 2016), which in western Volhynia is represented by such settlements as Holyshiv, Lutsk Raion and Lystvin, Dubno Raion (Rybicka 20I7: 53-59); however, there is no radiocarbon dating for them. For assemblages representing the late stages of the TC, such as Vynnyky-Zhupan, the Lviv Raion (Fig. I), Gordineşti and Hancauti, Edineț district, we now have a number of dates made of samples of good quality: cereals and animal bones coming from discrete features. They point to a period of about 3300-3000/2900 BC (Table I; Rybicka 2017: 133; Rybicka et al. 2019).

Also in materials from the late-beaker settlement in Zimne, some late Tripolyean traits, such as small globular amphorae or deep bowls painted with black paint, were identified, which Sławomir Kadrow, taking into account Taras Tkachuk's opinions, assigns to the Horodiştea and Gorodsk groups. He also wrote that the impact of this phase of the Tripolye culture appears in the form of "beaker" vessels with notches for the lid, bowls with a bevelled rim, and ornamented by an imprinted cord (Kadrow 2005: 13). Vessels with notches for the lid were also noted in Pawłosiów, site 52, located on the Rzeszów-Przemyśl loess areas, where they can be dated to 3500-3350 BC (Rybicka et al. 20I4: 193, table XXX: 2). Their presence was also noted in Piaski Wielkie in the Lublin region (Dobrzyński 20II: Fig. 9: 3, 6, IO: IO). Dates varying between 3600-3330 BC were obtained for two features from this site, and for one - a date of 3350-3100 BC (Dobrzyński 20II: 78). In the style of the pottery assemblage from Pawłosiów, site 52, Baden influences were also distinguished, in the form of the occurrence of single 
Chronology of the Funnel Beaker Culture Settlement in Western Ukraine ...

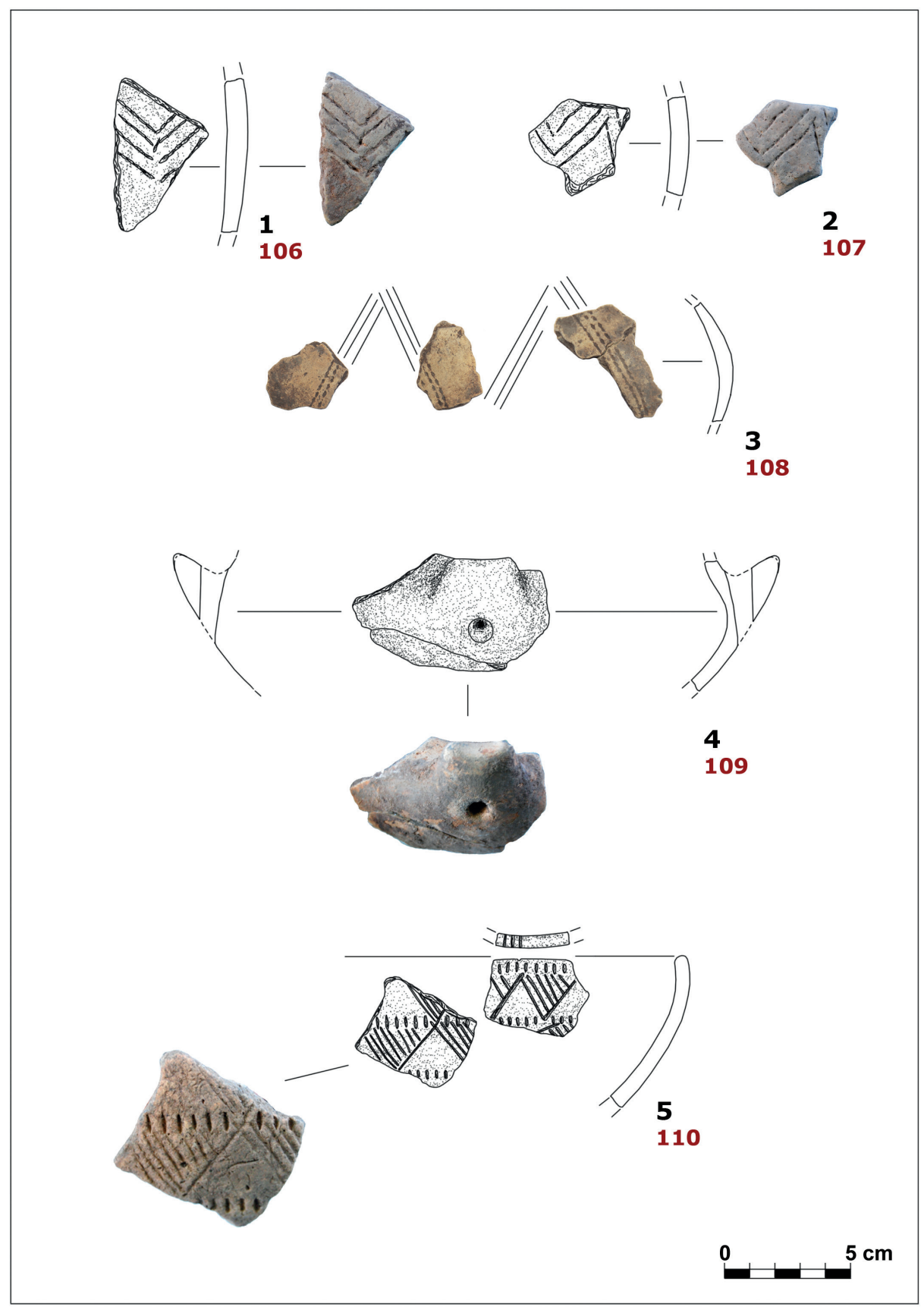

Fig. I. Zhupan site, Vynnyky near Lviv, pottery of the Tripolye culture. 
I08 $\mid$ Matgorzata Rybicka

\begin{tabular}{|c|c|c|c|c|c|c|c|c|c|c|}
\hline 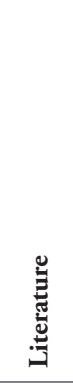 & 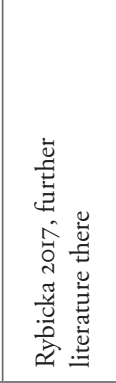 & 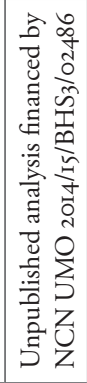 & 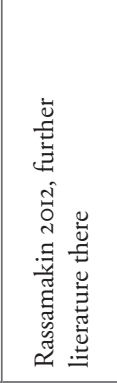 & 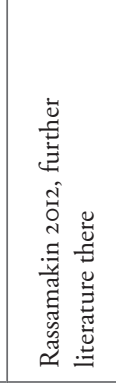 & 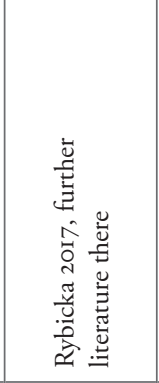 & 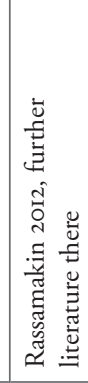 & 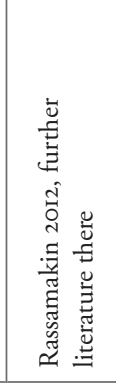 & 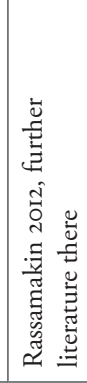 & 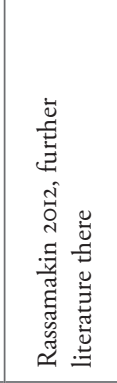 & 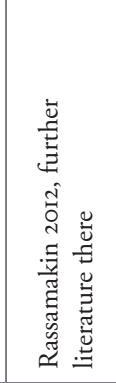 \\
\hline 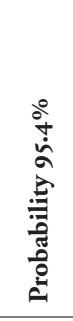 & 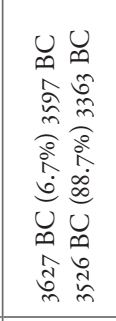 & 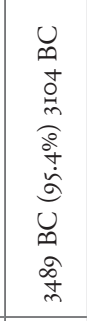 & 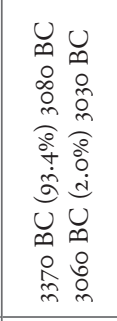 & 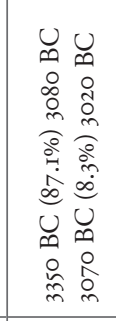 & 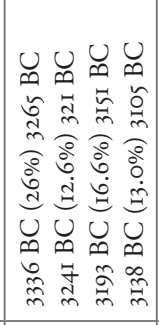 & 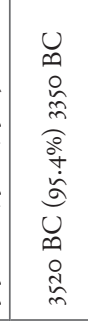 & 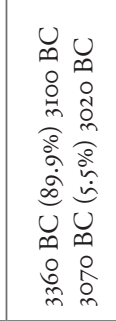 & 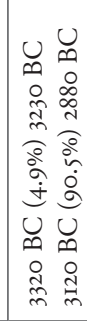 & 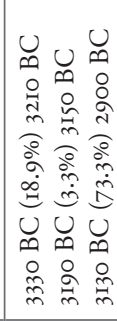 & 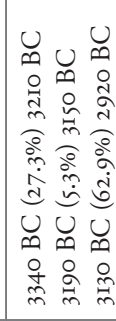 \\
\hline 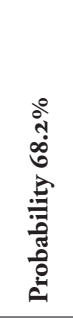 & 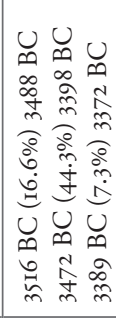 & 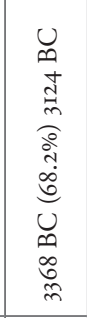 & 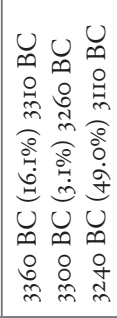 & 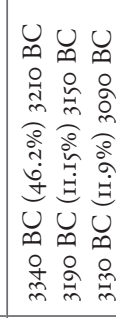 & 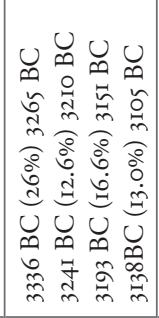 & 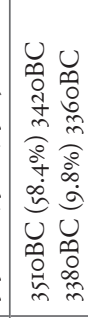 & 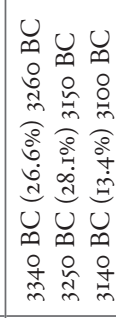 & 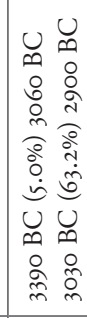 & 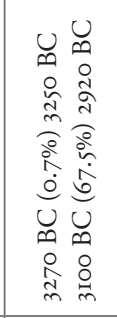 & 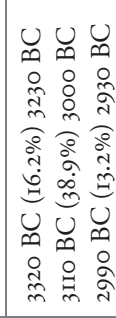 \\
\hline 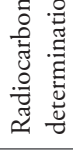 & 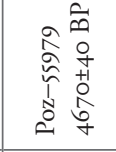 & 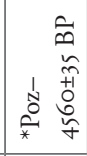 & 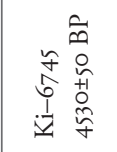 & 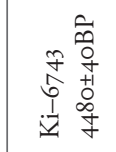 & 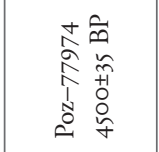 & 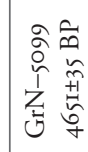 & 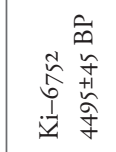 & 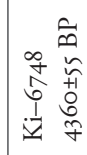 & 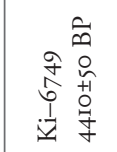 & 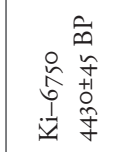 \\
\hline 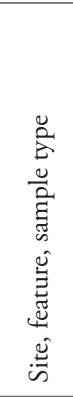 & 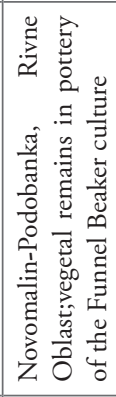 & 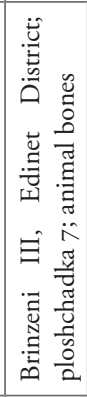 & 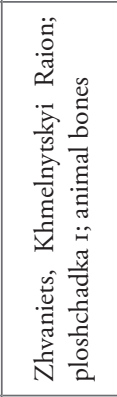 & 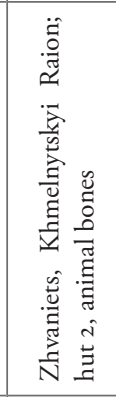 & 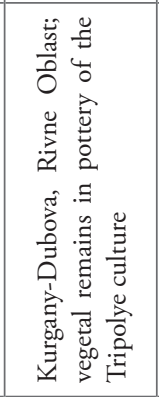 & 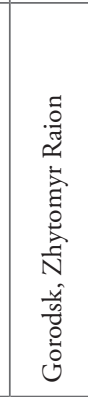 & 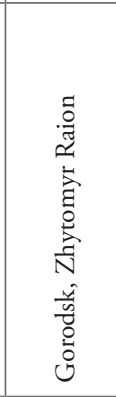 & 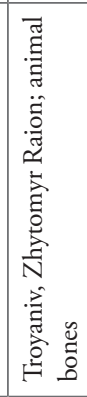 & 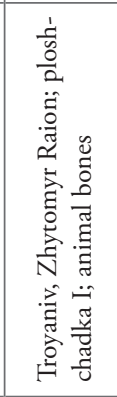 & 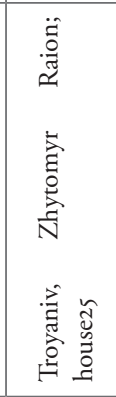 \\
\hline$\dot{\dot{z}}$ & $\dot{H}$ & $\dot{d}$ & $\dot{n}$ & $\dot{+}$ & $\dot{n}$ & $\omega^{\circ}$ & $\dot{\wedge}$ & $\infty$ & $\alpha$ & \\
\hline
\end{tabular}


Chronology of the Funnel Beaker Culture Settlement in Western Ukraine ...

\begin{tabular}{|c|c|c|c|c|c|c|c|c|c|c|}
\hline 莺 & 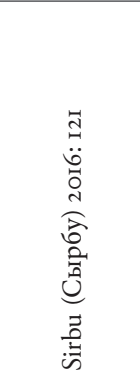 & 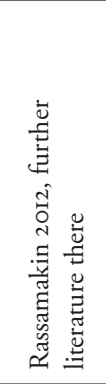 & 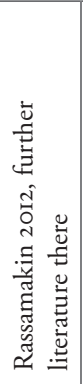 & 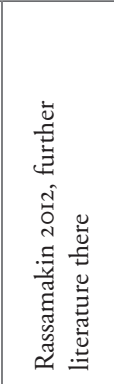 & 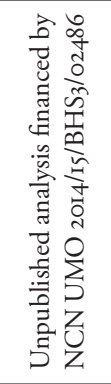 & 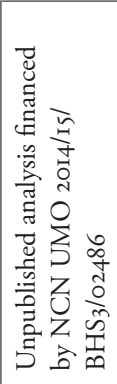 & 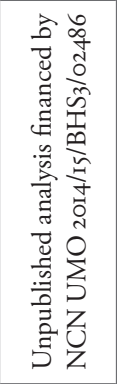 & 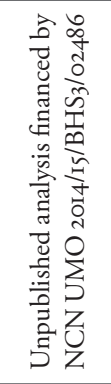 & 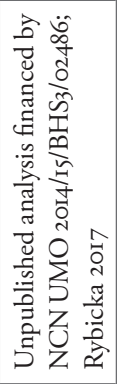 & 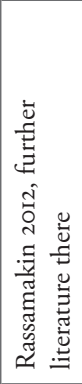 \\
\hline 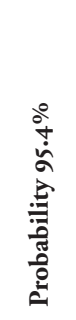 & 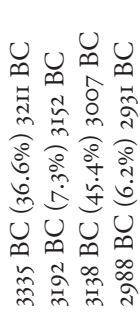 & 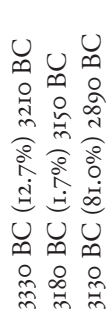 & 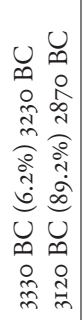 & 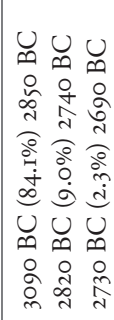 & 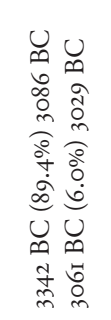 & 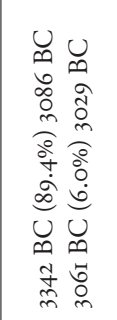 & 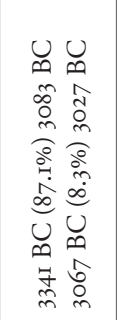 & 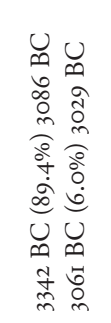 & 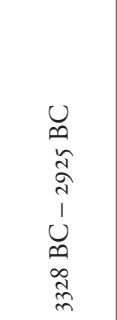 & 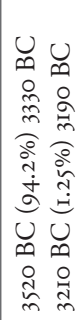 \\
\hline 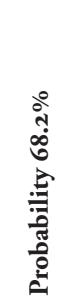 & & 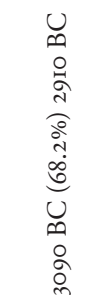 & 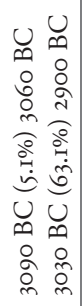 & $\begin{array}{l}0 \\
\infty \\
0 \\
0 \\
0 \\
0 \\
0 \\
i \\
\infty \\
0 \\
0 \\
0 \\
0 \\
0 \\
0 \\
0 \\
0\end{array}$ & 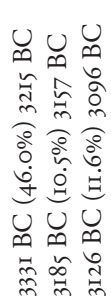 & 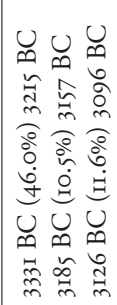 & 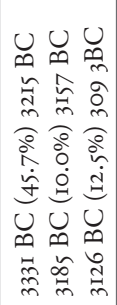 & 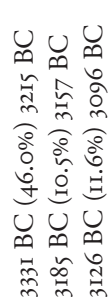 & 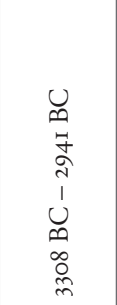 & 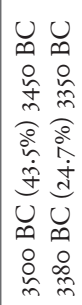 \\
\hline 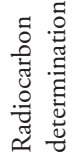 & 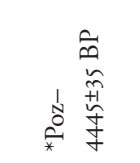 & 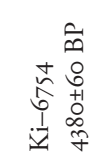 & 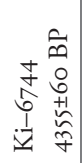 & 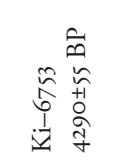 & 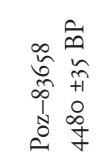 & 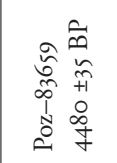 & 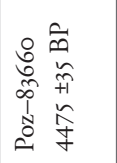 & 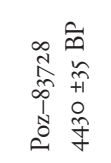 & 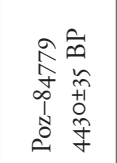 & 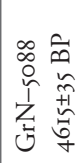 \\
\hline 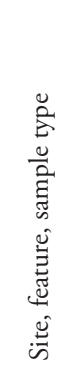 & 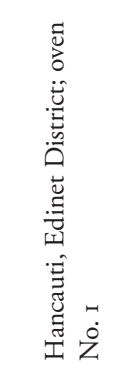 & 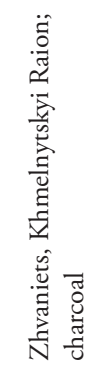 & 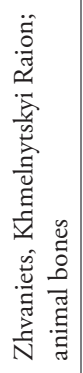 & 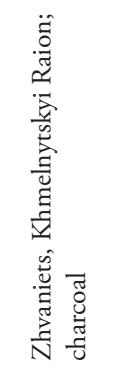 & 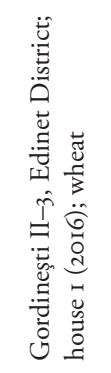 & 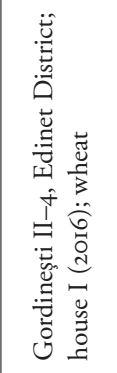 & 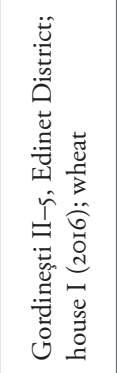 & 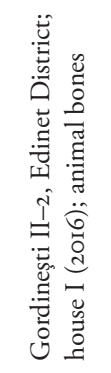 & 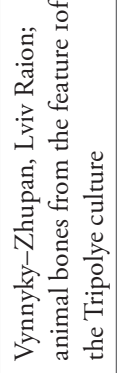 & 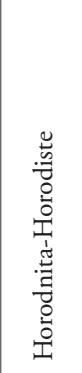 \\
\hline$z^{\circ}$ & $\dot{\Delta}$ & ḋ & $\ddot{n}$ & \pm & $\dot{\check{\sim}}$ & 7 & $\triangleq$ & $\infty$ & 9 & \\
\hline
\end{tabular}


IIo $\mid$ Matgorzata Rybicka

\begin{tabular}{|c|c|c|c|c|c|c|c|c|c|c|}
\hline 莺 & 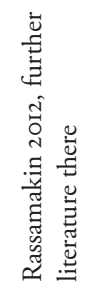 & 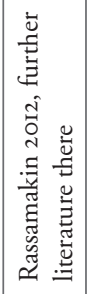 & 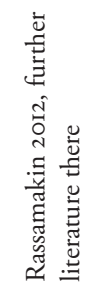 & 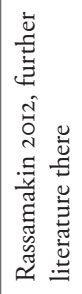 & 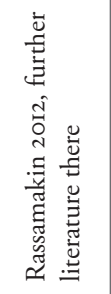 & 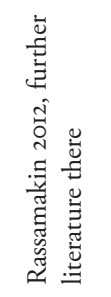 & 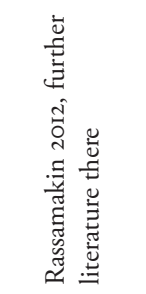 & 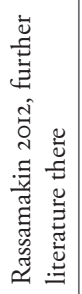 & 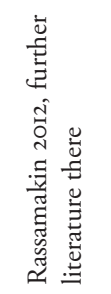 & 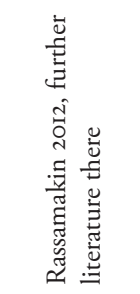 \\
\hline 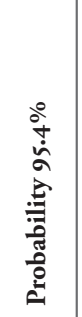 & 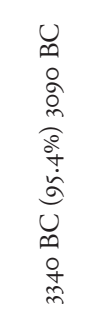 & 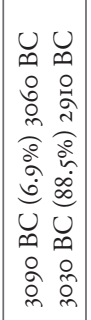 & 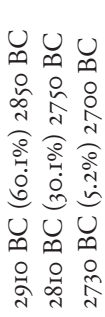 & 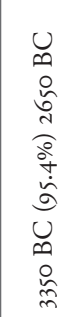 & 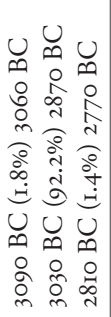 & 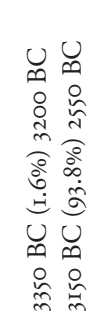 & 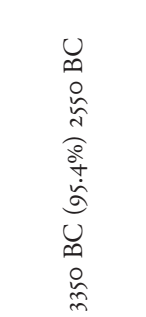 & 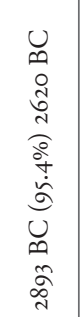 & 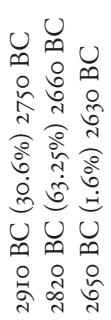 & 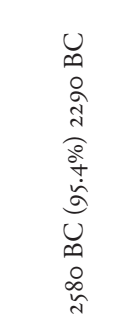 \\
\hline 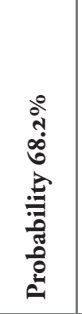 & 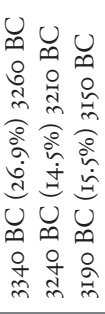 & 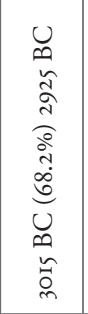 & 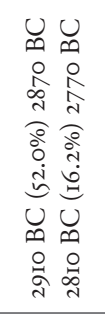 & 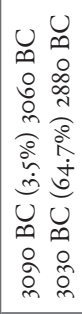 & 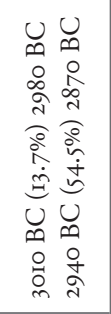 & 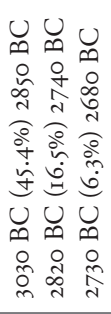 & 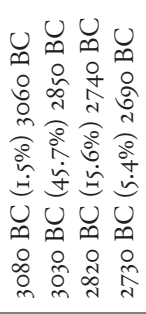 & 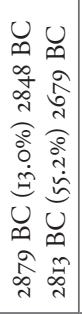 & 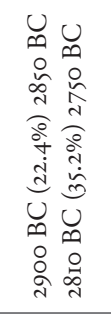 & 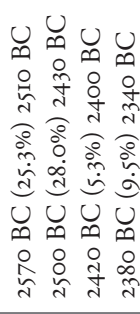 \\
\hline 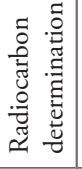 & 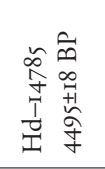 & 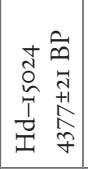 & 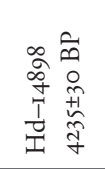 & 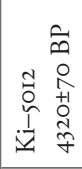 & 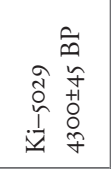 & 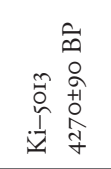 & 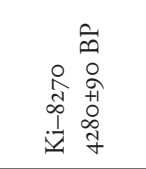 & 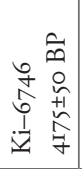 & 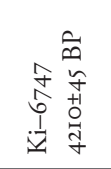 & 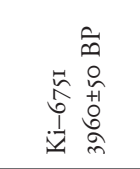 \\
\hline 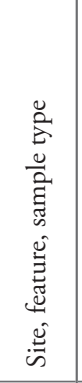 & 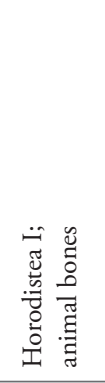 & 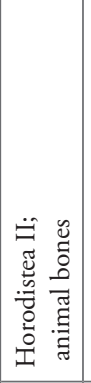 & 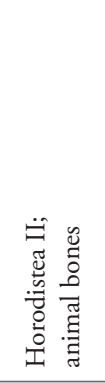 & 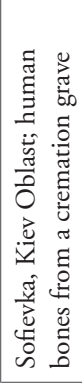 & 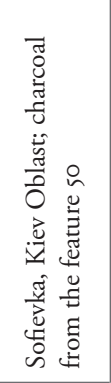 & 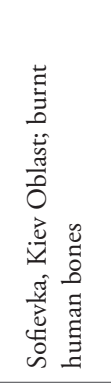 & 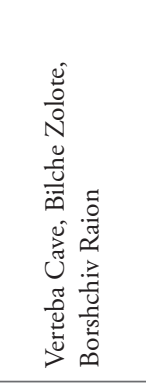 & 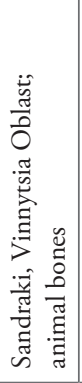 & 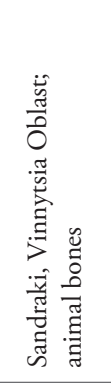 & 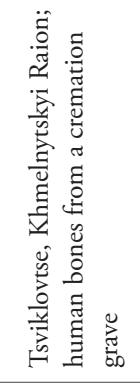 \\
\hline$\dot{z}$ & $\dot{\vec{H}}$ & त่ & $\dot{\tilde{d}}$ & A & สิ & 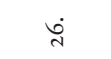 & ते & $\stackrel{\text { i }}{\circ}$ & ते & O \\
\hline
\end{tabular}


handles with knobs. Identical traits were also recorded in the materials of the Troyaniv and Gorodsk group (Videiko 2000), dated to the end of the fourth millennium BC (Table I). Sławomir Kadrow (2005: I4, Fig. I8, cf. Włodarczak 2006: 47-49) sees this type of decoration, having regard to the dating of the Sofievka group of the TC, as relating to influences from the Kostolac-Coţofeni-Cernavoda II and Sitagroi Va Radomir I-II - Junacite XIII-IX cultures. He does not consider that these include decorative inspiration from the northern Moldavian Gordineşti group of the TC, which is also characterised by pottery with similar stylistic features (Dergachev [Дергачев] 1980; Sirbu [Сырбу] 2016). It seems that the time of functioning of this group is now well defined and can be placed in the period 3300-3000/2900 BC (cf. Rybicka 20I7).

The pottery of the FBC originating from the pits No. 2/97 and 5/97 in Zimne (Bronicki et al. 2003) corresponds in terms of stylistics, for example, to ceramics from the Tshub site at Lezhnitsa, (Rybicka et al. 2019), but the dates obtained for these features raise doubts. In material from both mentioned settlements, in the methods of shaping rims and their edges (e.g. Bronicki et al. 2003: Figs II: II; I4: I5) have been noticed - distant analogies to the pottery morphology of the TC of Kurgany type from the Dubova site, Ostroh Raion (Verteletskyi [Вертелецкий] 2016), which is dated to the end-phase of the fourth millennium BC (Table I).

To sum up, the stylistics of the ceramics originating from some features from Zimne may be compared to the material from the site of Lysivka at Vynnyky, and from the Tshub site at Lezhnitsa (Rybicka et al. 2019), and chronologically probably corresponds to the second phase of the functioning of the settlement at Gródek.

\section{Grodzisko (Horodyshche) III site, Kotoryny, Zhydachiv Raion}

Also in the case of Kotoryny (Grodzisko [Horodyshche] III site), the situation in time of the settlement remains of the $\mathrm{FBC}$ is not unambiguous in the context of the radiocarbon determinations obtained and the identification there of the distinguishing features of the early-Funnel Beaker phase (cf. Hawinskyj et al. 2013; Rybicka 2016). The presence in a floor of features of numerous ceramic finds with traits corresponding to the style of the early stages of the eastern group of this culture justifies the acceptance of early radiocarbon determinations obtained for the material from pits in trench No. I,

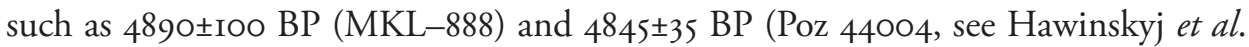
2013). The distinction in the ceramic material of references to the classical variety of the south-eastern group, such as handles of lunata type or curved rim profiles, also corresponds to the obtained ${ }_{14} \mathrm{C}$ determinations, and consequently makes possible dating of the first settlement phase of the FBC to the time range of 3650-3400 BC. The problem is, however, the cultural interpretation of several determinations representing the beginnings of the third millennium BC. A number of the distinguished features of the pottery decoration at Kotoryny, such as, for example, handles of lunata 
II2 $\mid$ Matgorzata Rybicka

type, Furchenstich, arcaded ornament, etc., were in use over a wide range in time (Włodarczak 2006: 52). However, there are also stylistic traits that can be assigned to the later stages of this culture, e.g. a wide Furchenstich and references to the style of the Baden Culture (Fig. 2). The so-called caterpillar stitch may be dated to a period of about 3400-3100 BC (Dobrzyński 20II: 76-78; Hawinskyj et al. 20I3: 266; Rybicka et al. 2019). The Baden elements present at Kotoryny can be assigned to the early stages of this cultural circle (cf. Fig. 2; Furholt 2009: I49-ISI). In this collection, however, there is neither late Tripolyean pottery nor its specific stylistic traits.

In the group of thirteen $\mathrm{I}_{4} \mathrm{C}$ determinations from Kotoryny, the majority (I2 dates) were made by scintillation from charcoal, and only one animal bone was dated by the AMS method. The oldest date: $5860 \pm 80$ BP (MKL-795), obtained for the pit No. 27, cannot be related to the FBC. It is probably the effect of dating secondarily deposited charcoals. Two consecutive dates were obtained for the base and top of the fill of this feature: $4630 \pm 90 \mathrm{BP}(\mathrm{MKL}-802)$ and $4620 \pm 70 \mathrm{BP}(\mathrm{MKL}-796)$, well correlating with the stylistic traits of the ceramics derived from it (Hawinskyj et al. 2013: 254). The dating resulting from analysis of animal bone from pit No. 26: 4845 \pm 35 BP (Poz-4404), corresponds to the result obtained for charcoals from pit No. 4: 4890 \pm IOO BP (MKL888). The results obtained are a positive verification of the dates derived from the stylistic characteristics of ceramics (Hawinskyj et al. 20I3: 235, 237). On the other hand, more disputable results came from samples from the post holes in trench II with the numbers: 26, 32 and 28, respectively: 5090 \pm I40 BP (MKL-884), $4690 \pm$ IIO BP (MKL-883) and $4240 \pm 90 \mathrm{BP}(\mathrm{MKL}-794)$. The large discrepancy between these dates makes their relationship with the FBC uncertain. The first two of them with a greater or lesser degree of probability match the dating of the ceramic stylistic traits from this trench, which are well represented by the material from the pit No. 27. The third result, however, does not correspond to the stylistic characteristics of these ceramics. For the shallow depression, referred to as pit No. 15 , two clearly different determinations were obtained: $5290 \pm 90 \mathrm{BP}(\mathrm{MKL}-885)$ and $4090 \pm 90 \mathrm{BP}(\mathrm{MKL}-892)$. In this case, one cannot determine what in fact they date. Such a large discrepancy means that they should be omitted in the assessment of the chronology of the remains of the FBC in Kotoryny. The same applies to the date of a charcoal sample from a shallow depression, the so-called pit No. 17: 4230 \pm 130 BP (MKL-889). However, the dating results obtained for a charcoal sample from the rampart and the pit No. 4a: 4520 770 BP (MKL-890) and $4420 \pm 90 \mathrm{BP}(\mathrm{MKL}-798)$ should be assigned to the settlement of Funnel Beaker times. It seems from the presented remarks that only a few determinations were made of samples from good contexts; these are the material from pits No. 4, 4a, 26, 27 as well as the layer under the rampart. The value of the others is questionable.

If we include only the radiocarbon dates from the sites in Kotoryny and Zimne, which correspond to the accepted stylistic dating of the ceramics derived from them and representing good contexts, the FBC in Western Ukraine can be dated to the period 
Chronology of the Funnel Beaker Culture Settlement in Western Ukraine ...
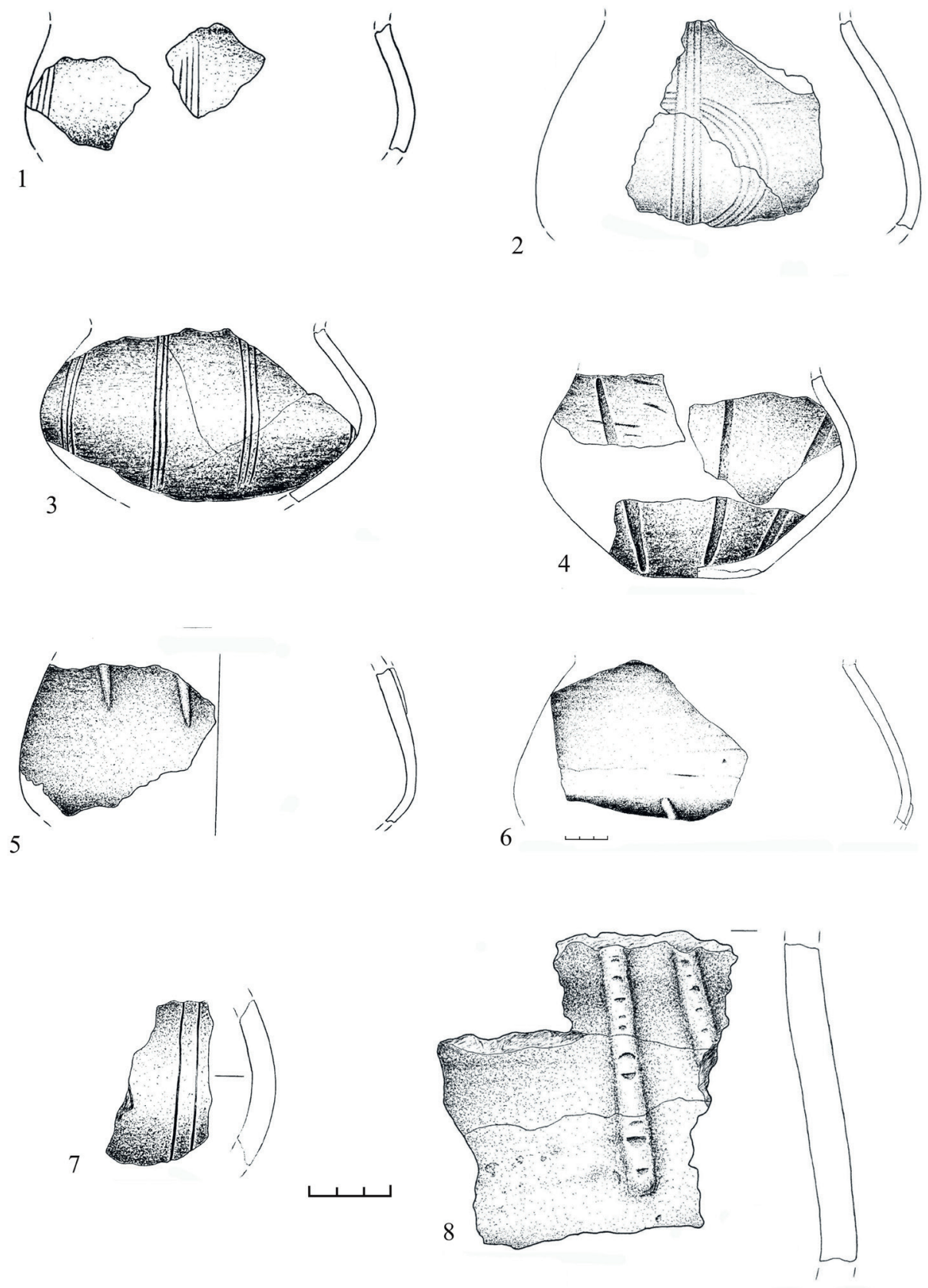

Fig. 2. Kotoryny near Zhydachiv, Grodzisko (Horodyshche) III site. Pottery of the Funnel Beaker culture. 
II4 $\mid$ Matgorzata Rybicka

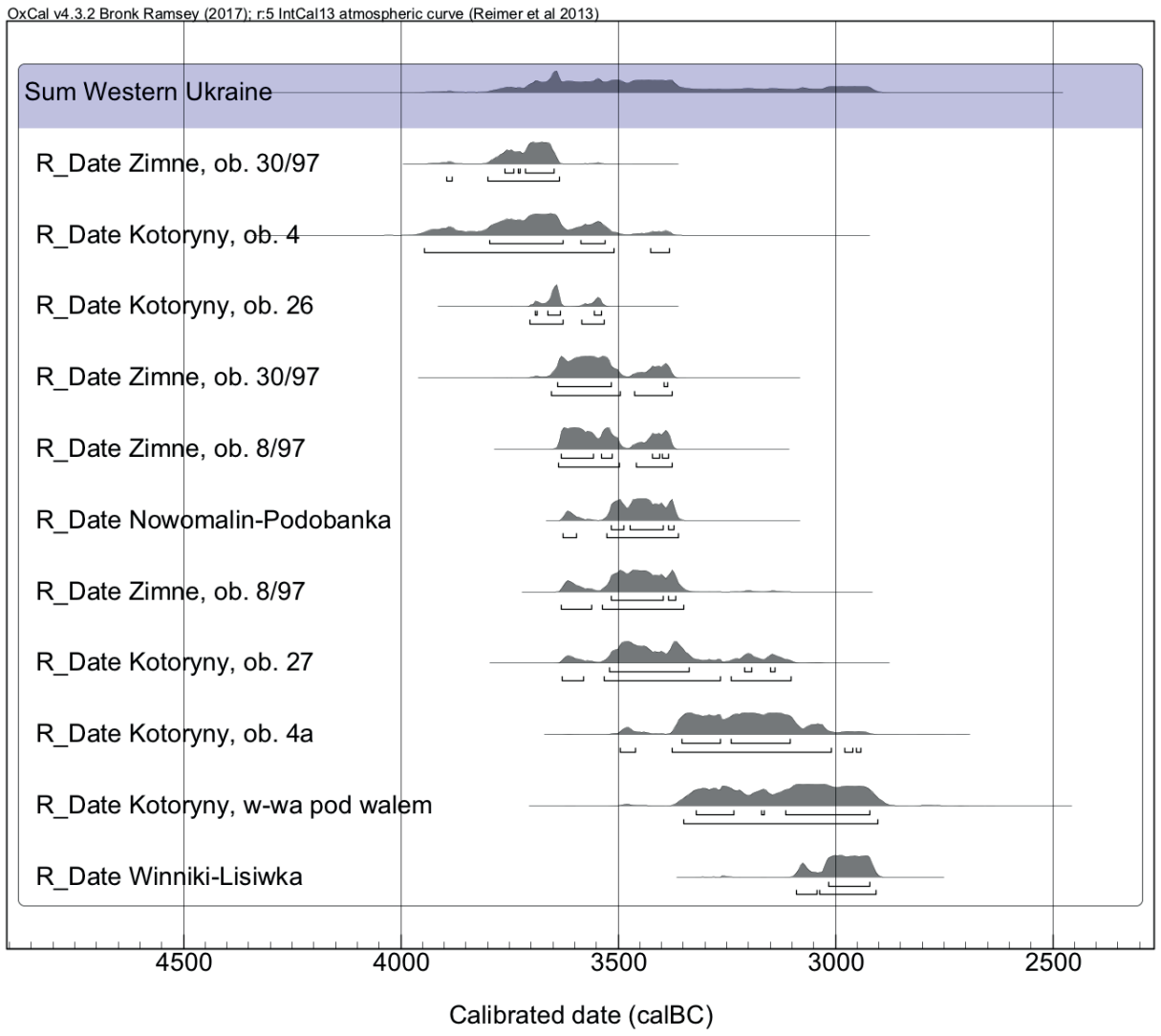

Fig. 3. Dating of the Funnel Beaker culture in Western Ukraine.

$3700 / 3600-3000 / 2900$ BC (Fig. 3). Generally, this corresponds to the chronology of the settlement in Gródek (Włodarczak 2006), and the Gordineşti group could be also included in it (Table I). The presence of traits representing this culture both in Gródek (Rybicka 20I7) and in Zimne (Kadrow 2005) confirms the above presented suggestion.

\section{CONCLUSIONS}

The dates obtained for Gródek and cited by Jan Kowalczyk (1969) as justifying the very early emergence of the $\mathrm{FBC}$ in the Bug River region, have been subjected to verification (Bronicki et al. 2003: 3I; Włodarczak 2006: 33). The repeated analysis of one of these samples resulted in a much later result, which corresponds to the date 
range of the classical stage of the south-eastern group of this culture, ascertained for assemblages from Bronocice (Kruk and Milisauskas 20I8). Dates associated with the early-beaker stylistics at Kotoryny (Grodzisko [Horodyshche] III site) are not conformable with the concept of Jan Kowalczyk (1969) regarding the chronology of the initiation of this culture in western Ukraine. However, they justify, just like the stylistics of the ceramics, the emergence of the FBC in this area at a similar time as in the Rzeszów-Przemyśl loess areas (cf. Rybicka 20I6). The problem is, however, to determine the date of its disappearance, both in the Bug region and on the upper Dniester. An unequivocal explanation of this issue requires conducting more radiocarbon analyses for samples of "short-lived" materials coming from homogeneous contexts (e.g. cereals, animal bones). At present, it seems that the disappearance of the FBC in those regions can be assigned to the turn from the fourth to the third millennium BC. This is indicated by the presence of imports from the Gordineşti group at Gródek (Gumiński 1989; Włodarczak 2006) and Zimne (Kadrow 2005).

Translated by Andrzej Leligdowicz

\section{REFERENCES}

Balcer, B. 198I. Związki między kulturą pucharów lejkowatych (KPL) a kulturą trypolską (KT) na podstawie materiałów krzemiennych. In T. Wiślański (ed.), Kultura pucharów lejkowatych $w$ Polsce (studia i materiaty), 8I-9I. Poznań.

Bronicki, A., Kadrow, S. and Zakościelna, A. 2003. Radiocarbon Dating of the Neolithic Settlement in Zimne, Volhynia, in Light of the Chronology of the Lublin-Volhynia Culture and the SouthEastern Group of the Funnel Beaker Culture. In A. Kośko (ed.), The Foundations of Radiocarbon Chronology of Cultures between the Vistula and Dnieper: 4000-I00o BC, 22-66. Poznań. Baltic-Pontic Studies I2.

Dergachev, V. (Дергачев, В.) 1980. Pamyatniki pozdnego Tripolya. Kischinev [Памятники позднего Триполья. Кишинев].

Diachenko, A., Rybicka, M., Król, D. and Sîrbu, G. 2019. Between the East and the West. Dynamics of Social Changes from the Eastern Carpathians to the Dnieper in the 4th-beginning of 3 rd Millennium BC (Preliminary study). Rzeszów.

Dobrzyński, M. 20II. Dom i otoczenie na podstawie osady kultury pucharów lejkowatych w Piaskach Wielkich, pow. Świdnik, woj. lubelskie. In M. Rybicka (ed.), Obraz struktury spotecznej w świetle źródet archeologicznych w pradziejach i średniowieczu, 67-8I. Rzeszów.

Furholt, M. 2009. Die nördlichen Badener Keramikstile im Kontext des mitteleuropäischen Spätneolithikums (3650-2900 v. Chr.). Bonn. Studien zur Archäologie in Ostmitteleuropa 3.

Gumiński, W. 1989. Gródek Nadbużny. Osada kultury pucharów lejkowatych. Wrocław-Warszawa-KrakówGdańsk-Łódź. Polskie Badania Archeologiczne 28.

Havinskyi, А. (Гавінський А.) 2009. Poselenniya kulturi lyichastogo posudu v Malih Hribovichah. Materialy $i$ doslidzhennya $z$ arheologyi Prykarpattya $i$ Volyni: 172-197 [Поселення культури 
II6 $\mid$ Matgorzata Rybicka

лійчастого посуду в Малих Грибовичах. Матеріали і Дослидження з Археологї Прикарпаття і Волині г3: 172-197].

Havinskyi, A. and Pasterkevich, V. (Гавінський, А. and Пастеркєвіч, В.). 2ог6. Arheologichni pamyatki kulturi lyichastogo posudu na teritoriy Ukrayni. Lviv [Ареологічні памятки культури лійчастого посуду на території України. Львів].

Hawinskyj, A., Pasterkiewicz, W. and Rybicka, M. 20I3. Kotoryny, rej. Żydacziw, stan. Grodzisko III. Osadnictwo z okresu neolitu. In K. Harmatowa, J. Machnik and M. Rybicka (eds), Natural environment and man on the upper Dnister - Region of the Halyč-Bukacivci Basin - in prehistory and early medieval period, 169-284. Kraków.

Kadrow, S. 1991. Iwanowice, stanowisko Babia Góra. Część I: Rozwój przestrzenny osady z wczesnego okresu epoki brązu. Kraków.

Kadrow, S. 2005. Związki kultury trypolskiej z kulturami środkowej i południowo-wschodniej Europy. In A. Garbacz and M. Kuraś (eds), Kultura trypolska. Wybrane problemy, 7-31. Stalowa Wola.

Kowalczyk, J. 1968. Dwa zespoły radiowęglowe datowane radiowęglem. Wiadomości Archeologiczne 33: 368-376.

Kowalczyk, J. 1969. Początki neolitu na ziemiach polskich. Wiadomości Archeologiczne 34(I): 3-69.

Kośko, A. 1981. Udziat potudniowo-wschodnioeuropejskich wzorców kulturowych w rozwoju niżowych spoteczeństw kultury pucharów lejkowatych. Grupa mątewska. Poznań. Uniwersytet im. Adama Mickiewicza w Poznaniu. Seria Archeologiczna 19.

Kruk, J. and Milisauskas, S. 20I8. Bronocice. The Chronology and Development of a Neolithic Settlement of the Fourth Millennium BC. Kraków.

Movsha, Т. (Мовша, T.) 1985. Vzaemovidnosini Tripillya-Kukuteni z sinhronnimi kulturami Tsentralnoj Evropi. Arheologiya 51: 22-31 [Взаемовідносини Трипілля-Кукутені з синхронними культурами Центральної Європи. Археологія 5г: 22-31].

Nowak, M. 2009. Drugi etap neolityzacji ziem polskich. Kraków.

Peleshchyshyn, М. (Пелещишин, М.) I990. Plemena kulturi voronkovidnih sosudov. In A. P. Chernysh (ed.), Arheologiya Prikarpattya, Volyni i Zakarpattya (eneolit, bronza i rannee zhelezo), 35-43. Kiev [Племена культуры воронковидных сосудов. In А. П. Черныш (ed.), Археология Прикарпатья, Вольни и Закарпатья (енеолит, бронза и раннее железо), 35-43. Киев].

Peleshchyshyn, М. (Пелещишин, М.). 2004. Eneolitichne poseleniya Zimne v Zahidniy Volini. Ternopil [Енеолітичне поселення Зимне в Західній Волині. Тернопіль].

Rassamakin, Y. 20I2. Absolute chronology of Ukrainian Tripolian settlement. In F. Menotti and A. G. Korwin-Piotrowskiy (eds), The Tripolye Culture Giant-Settlements in Ukraine. Formation, Development and Decline, 19-69. Oxford.

Rybicka, M. 20I6. Nowe dane w kwestii datowania kultury pucharów lejkowatych na Podgórzu Rzeszowskim. Materiaty i Sprawozdania Rzeszowskiego Ośrodka Archeologicznego 37: 69-83.

Rybicka, M. 2017. Kultura trypolska - kultura pucharów lejkowatych. Natężenie kontaktów i ich chronologia. Rzeszów. Collectio Archaeologica Ressoviensis 37.

Rybicka, M., Hawinskyj, A. and Pasterkiewicz, W. 2019. Leżnica, stanowisko Czub-osiedle kultury pucharów lejkowatych na zachodnim Wotyniu (z analizą zabytków krzemiennych autorstwa Witalija Konopli). Rzeszów.

Rybicka, M., Król, D. and Rogoziński, J. 20I4. Pawłosiów, stan. 52, woj. podkarpackie. Osada kultury pucharów lejkowatych. In M. Rybicka (ed.), Wschodnie pogranicze grupy potudniowo-wschodniej kultury pucharów lejkowatych, 67-256. Rzeszów. Collectio Archaeologica Ressoviensis 28.

Sirbu, G. (Сырбу, Г.) 2016. Nekotoriye idei otnositelno hronologii pozdne eneoliticheskoy gruppy Gordinesht v pruto-dnestrovskom mezhdureche. [Некоторые идеи относительно хронологии поздне энеолитической группы Гординешть в пруто-днестровском междуречье]. In 
M. Rybicka and N. Biłas (eds), Datowanie-Chronologia-Periodyzacja. Różne aspekty czasu w archeologii, I08-II6. Lviv. Naukovi studyi 9 [Львів. Наукоbi ctydii 7].

Verteletskyi, D. (Вертелецкий, Д.) 20I6. Keramika piznotripolskogo horizontu z poselenniya KurhanyDubova [Керамика пізньотрипольского горизонту з поселенния Курганы-Дубова]. In A. Diaczenko, D. Król, A. Kyrylenko, M. Rybicka and D. Werteletski (eds), NowomalinPodobanka i Kurgany-Dubowa. Osiedla kultury trypolskiej na zachodnim Wotyniu, 7I-IOO. Rzeszów.

Videiko, M. 2000. Tripolye and the cultures of central Europe: facts and character of interactions: 4200-2750 BC. In A. Kośko (ed.), The Western Border Area of the Tripolye culture, 13-68. Poznań. Baltic-Pontic Studies 9.

Włodarczak, P. 2006. Chronologia grupy południowo-wschodniej kultury pucharów lejkowatych w świetle dat radiowęglowych. In J. Libera and K. Tunia (eds), Idea megalityczna w obrządku pogrzebowym kultury pucharów lejkowatych, 27-66. Lublin-Kraków.

Zaharuk, Yu. (Захарук, Ю.) 1959. Do pitannya pro spivvidnoshennya i zv'yazky mizh kulturoyu lyichastogo posudu ta tripilskogo kulturoyu. Materialy $i$ doslidzhennya $z$ arheologyi Prykarpattya $i$ Volyni: 54-72 [До питання про співвідношення і зв ' язки між культурою лійчастого посуду та трипільською культурою. Матеріали і дослідження з археології Прикарпатття і Волині: $54-72]$.

Zbenovich, V. (Збенович, В.) I976. Pozdneye Tripolye I ego svyazi s kulturami Prikarpatya i Malopolshi [Позднее Триполье и его связи с культурами Прикарпатья и Малопольши]. Acta Archaeologica Carpathica I6: 2I-58. 
